\title{
PERFIL DE USUARIOS DE UNA GLÍNIGA UNIVERSITARIA DURANTE EL ESTADO DE ALARMA SANITARIA GOVID 19
}

\author{
USER PROFILE IN AN UNIVERSITY GLINIC \\ DURING THE COVID-19 HEALTH ALARM
}

\author{
PURIFICACIÓN SIERRA-GARCÍA ${ }^{1}$, \\ LUIS ÁNGEL SAÚL ${ }^{1}$, MigUEL ÁNGEL CARRASCO ${ }^{1} \mathrm{Y}$ \\ MARCELA PAZ GONZÁLEZ-BRIGNARDELLO ${ }^{1}$
}

Cómo referenciar este artículo/How to reference this article:

Sierra-García, P., Saúl, L. Á., Carrasco, M. Á. y González-Brignardello, M. P. (2021). Perfil de usuarios de una clínica universitaria durante el estado de alarma sanitaria COVID 19 [User Profile in an University Clinic during the COVID-19 Health Alarm]. Acción Psicológica, 18(1), 11-26. https://doi.org/10.5944/ap.18.1.29867

\section{Resumen}

El presente estudio pretende analizar el perfil de los usuarios atendidos por el dispositivo especial de atención psicológica en línea habilitado por el Servicio de Psicología Aplicada (SPA) durante el primer período de alerta sanitaria COVID-19. La muestra, compuesta por 124 participantes $(70.4 \%$ mujeres $)$ con una edad promedio de 41.49 años cumplimentó las versiones abreviadas del Listado de síntomas (SA-45), la medida de resultados clínicos (CORE-OM), el Inventario de afrontamiento (COPE-28) y la Escala de resiliencia de Connor-Davidson (CD-RISC). Los resultados mostraron un perfil sociodemográfico formado mayoritariamente por mujeres estudiantes o del sector servicios, solteras y de nacionalidad española, con problemas previos de salud mental. Congruentemente con los motivos de consulta, mostraron predominantemente sintomatología de depresión, ansiedad y somatización, un deficiente uso de estrategias de afrontamiento y bajos niveles de resiliencia. De las variables mencionadas puede extraerse un posible

Correspondence address [Dirección para correspondencia]: Miguel Ángel Carrasco, Facultad de Psicología, Universidad Nacional de Educación a Distancia, España.

Email: mcarrasco@psi.uned.es

ORCID: Purificación Sierra García (https://orcid.org/0000-0003-3774-2975), Luis Ángel Saúl (https://orcid.org/00000002-6351-8283), Miguel Ángel Carrasco (https://orcid.org/0000-0003-3282-818X) y Marcela Paz González Brignardello (https://orcid.org/0000-0001-7113-7846).

${ }^{1}$ Universidad Nacional de Educación a Distancia, España.

Recibido: 2 de abril de 2021.

Aceptado: 8 de junio de 2021 . 
perfil de riesgo en población clínica que perfila un grupo vulnerable sobre el que se precisan medidas de prevención e intervención psicológicas.

Palabras clave: COVID-19; impacto psicológico; factores de riesgo; afrontamiento; resiliencia.

\section{Abstract}

This study aims to analyze the profile of users in an university clinic (SPA-UNED) during the first COVID-19 19 health alarm. The sample was composed of 124 participants ( $70.4 \%$ women) with an average age of 41.49 years and four instruments were used: Symptom Assessment-45 Questionnaire (SA-45), Clinical Outcomes in Routine Evaluation-Outcome Measure (CORE-OM), Coping Orientation to Problems Experienced (COPE-28) and the Scale Connor-Davidson Resilience (CD-RISC). The results showed a sociodemographic profile formed mainly by single Spanish women from the service sector. Consistent with the reasons for consultation, participants predominantly showed symptoms of depression, anxiety, and somatization, poor use of coping strategies, and low levels of resilience. These variables may be a possible risk profile in the clinical population that outlines a vulnerable group for which psychological prevention and intervention measures are required.

Keywords: COVID-19; psychological impact; risk factors; coping; resilience.

\section{Introducción}

La magnitud de la pandemia mundial causada por la COVID-19 alcanza ya, al año de su aparición en China, los 92 millones de personas infectadas y dos millones de muertos, con 215 países afectados (Organización Mundial de la Salud, 2021). Los intentos de control de la pandemia han llevado a los gobiernos a implementar estrictas medidas restrictivas que incluyen períodos de confinamiento y

\footnotetext{
1 Para precisión terminológica ver Sánchez-Villena y De la Fuente-Figuerola (2020), y Wilder-Smith y Freedman (2020).
}

cuarentenas prolongados, restricciones a la movilidad, cierre de fronteras, distancia social, entre otras. Son de especial interés las consecuencias psicológicas y sobre la salud mental que esta situación ha provocado, así como la evolución que esos efectos tienen, a medio y largo plazo.

A partir de otras situaciones previas de pandemia ocurridas en las últimas dos décadas (i.e., SARS, MERS, $\mathrm{H} 1 \mathrm{~N} 1$ y ébola) y de los primeros estudios publicados durante esta, podemos identificar los principales factores específicos que parecen impactar la salud mental: (a) la vivencia prolongada e incluso reiterada de cuarentena o confinamiento $^{1}$ (e.g., Brooks et al., 2020) y las restricciones a la movilidad (Cabrera, 2020), el aislamiento y la soledad (Killgore et al., 2020); (b) la sobrecarga laboral y emocional, especialmente de los profesionales sanitarios de primera línea y su exposición permanente al riesgo de infección, estigma y miedo a transmitir la enfermedad a los familiares (Conversano et al., 2020); (c) la infección y las sucesivas etapas del desarrollo de la enfermedad, las vivencias intra y extrahospitalarias, permanencia en las unidades de cuidados intensivos (UCIs), riesgo vital, etc. (e.g., Taquet et al., 2021; Wu et al., 2005); (d) el proceso de duelo complejo, la imposibilidad de acompañar y despedir al ser querido, así como la falta de rituales sociales que proveen de apoyo social (Araujo-Hernández et al., 2020; Mortazavi et al., 2020; Wallace et al., 2020); (e) la exposición repetida a los medios de comunicación (Garfin et al., 2020); (f) las consecuencias económicas y laborales, así como la preocupación por la crisis económica venidera (Rodríguez-Rey et al., 2020); y (g) los posibles efectos biológicos directos (e.g., afectación del SNC, alteración de estados de conciencia, etc.) relacionados con la infección vírica o su tratamiento (Kim y Su, 2020; Szcześniak et al., 2021; Taquet et al., 2021).

Los primeros estudios sobre el impacto psicológico que la situación ha provocado en los seis meses iniciales tras la pandemia refieren altas tasas de estrés, ansiedad, depresión, frustración e incertidumbre (Serafini et al., 2020); también miedos e insomnio (Sandín et al., 2020), reacciones de negación, ira y miedo (Torales et al., 2020); trastorno de estrés postraumático (North et al., 2021); al- 
teraciones del sueño (Casagrande et al., 2020) y abuso de sustancias (Czeisler et al., 2020), principalmente. Estudios a gran escala muestran que los trastornos de ansiedad y los síntomas depresivos son los más prevalentes a lo largo de las diferentes etapas de la pandemia, con una frecuencia informada que oscila entre el $20-25 \%$ y el $50 \%$ (Fancourt et al., 2021; Qiu et al., 2020). En Reino Unido, una encuesta nacional realizada a los tres meses del comienzo de la pandemia, encontró que casi el $30 \%$ de los encuestados cumplían el punto de corte para considerar la presencia de un trastorno psiquiátrico general, con síntomas depresivos, de ansiedad y pérdida de confianza, entre otros (Li y Wang, 2020). En España, tres estudios analizan las consecuencias psicológicas ocurridas durante los meses de marzo y abril del 2020: (a) Rodríquez-Rey et al. (2020) mostraron que cerca del $36 \%$ de los sujetos refirieron estar sufriendo impacto psicológico de grado moderado a severo, el $25 \%$ niveles de ansiedad entre leve y severos, $41 \%$ presentó síntomas depresivos y el $41 \%$ refirió sentirse estresado, (b) Sandín et al. (2020) informan que las experiencias emocionales más frecuentemente referidas fueron: preocupación (49.9\%), estrés $(45.5 \%)$, desesperanza $(44.9 \%)$, depresión $(39.6 \%)$ y problemas de sueño (43.3 \%); y (c) Balluerka et al. (2020) reportaron de incremento en el malestar psicológico de la siguiente manera: Incertidumbre $(84.4 \%)$, miedo a perder seres queridos $(82.5 \%)$, preocupación por contraer enfermedades graves, incluida la COVID-19 (71\%), problemas del sueño $(61.4 \%)$, sentimientos depresivos $(57 \%)$, irritación/enfado (56\%), agobio por el número de mensajes (53\%), cambios de humor (53\%), dificultades para concentrarse $(52 \%)$ y sentimientos de irrealidad $(50.2 \%)$.

En Italia, al comienzo de la pandemia, casi la mitad de los encuestados reportaron disminución de bienestar psicológico, y un $5 \%$ refirió sintomatología de TEPT (Favieri et al., 2020); Casagrande et al. (2020) informaron que el $57.1 \%$ presentó deterioro del sueño, el $32.1 \%$ alta ansiedad, el $41.8 \%$ alto distrés y el $7.6 \%$ síntomas de TEPT. Muchas de las respuestas tempranas son adaptativas frente a la situación excepcional vivida. En parelelo, Fancourt et al. (2021) en Reino Unido encontraron que la sintomatología ansiosa y depresiva presente en la primera semana disminuyó durante las siguientes semanas de restricción estricta, estabilizándose cuando se aliviaron las medidas. Sin embargo, otros estudios muestran un aumento de sin- tomatología a lo largo de las semanas de confinamiento (Ozamiz-Etxebarria et al., 2020).

Los factores de riesgo asociados al desarrollo de trastornos mentales son: ser personal sanitario de primera línea (Dosil Santamaría et al., 2020), ser mujer o joven (Casagrande et al., 2020; Favieri et al., 2020; Qiu et al., 2020; Rodríguez-Rey et al., 2020), tener o haber tenido diagnóstico de COVID-19 (Li y Wang, 2020; Taquet et al., 2021), tener incertidumbre de haber sido expuestos al virus (Favieri et al., 2020), tener enfermedad mental previa (Mak et al., 2009), haber perdido el trabajo (Rodríguez-Rey et al., 2020) o tener bajo nivel educativo y bajo nivel de ingresos (Fancourt et al., 2021). Por otra parte, se han identificado algunos factores protectores, entre los cuales se encuentra vivir en pareja y tener trabajo (Li y Wang, 2020).

El presente artículo tiene como objetivo analizar el perfil de los usuarios atendidos por el dispositivo gratuito especial de atención psicológica online, mediante teléfono y videoconferencias y habilitado por el Servicio de Psicología Aplicada (SPA) durante el primer período de alerta sanitaria COVID-19 en el que se produjo un estado de confinamiento generalizado en la población española durante, aproximadamente, cuatro meses. Más específicamente, se pretendió mediante este estudio: (a) analizar el perfil sociodemográfico, las principales demandas y los antecedentes de las personas que solicitaron ayuda durante el confinamiento COVID-19; (b) explorar la sintomatología y significación clínica de estos usuarios; (c) estudiar sus estrategias de afrontamiento y nivel de resiliencia; y finalmente (d) analizar las diferencias por sexo en cada uno de los objetivos anteriores.

\section{Método}

\section{Participantes}

Los participantes que componen la muestra de este estudio fueron los usuarios atendidos por el Servicio de Psicología Aplicada (SPA) de la Universidad Nacional de Educación a Distancia (UNED), dentro del dispositivo de atención psicológica activado con motivo del confinamiento derivado del estado de alarma por la COVID-19 y 
el cual se extendió entre los meses de marzo a junio del año 2020. Se ofreció participar a todas aquellas personas que demandaron atención psicológica en ese periodo para el dispositivo especial. El servicio de atención psicológica para los usuarios habituales quedó suspendido temporalmente. Se trata de una muestra incidental, no aleatoria, reclutada de entre aquellas personas que hicieron uso de dicho dispositivo y que aceptaron voluntariamente participar en este estudio.

El número total de participantes fue de $124(70.4 \%$ mujeres) con una edad promedio de 41.49 años (Desviación tipo $=16.60$ ) que osciló en un rango de 24 a 58 años. Del conjunto de participantes, una submuestra equivalente ( $n=60 ; 70.4 \%$ mujeres) cumplimentó varios cuestionarios relativos a síntomas psicopatológicos y estrategias de afrontamiento. El resto desistió en participar en la cumplimentación de los cuestionarios mencionados.

Una información detallada sobre las características de la muestra se presenta en la Tabla 1 correspondiente al apartado de resultados.

\section{Instrumentos de evaluación}

Registro ad hoc de datos diseñado por el SPA. El registro consistía en una ficha de diferentes datos sobre el usuario que cumplimentaban los psicólogos que atendían a los pacientes. Los datos a cumplimentar incluyeron: datos sociodemográficos, motivo de consulta, antecedentes familiares y personales relacionados con la demanda.

Listado de síntomas SCL-90 de Derogatis. Versión abreviada SA-45 (Sandín et al., 2008). Este instrumento es la adaptación del Symptom Assessment-45 Questionnaire (SA-45) de Davison et al. (1997). Es un instrumento de autoinforme de síntomas psicopatológicos de 45 ítems derivado del SCL-90 (Derogatis, 1977). Para este estudio se utilizó la versión española validada por Sandín, et al. (2008). Consiste en nueve escalas de 5 ítems cada una, que evalúan las mismas dimensiones que el SCL-90: hostilidad, sensibilidad interpersonal, somatización, ansiedad, psicoticismo, obsesión-compulsión, ansiedad fóbica e ideación paranoide. Los participantes deben indicar cuánto ha estado presente cada uno de los 45 síntomas durante la última semana según una escala Likert de cinco puntos que va desde 0 («Nada en absoluto) hasta 4 («Mucho o extremadamente»). El cuestionario presenta propiedades psicométricas adecuadas tanto en lo que concierne a la fiabilidad (coeficientes alfa de Cronbach y correlaciones ítem escala) como a la validez.. A esta versión abreviada se añadieron tres ítems adicionales: dos referidos a sintomatología rumiativa (i.e., "Siento preocupación y ansiedad por muchas cosas y no puedo controlarlo"; "Me preocupo en exceso por muchas cosas como la salud, familia, economía, trabajo, amigos... y no puedo controlarlo") y uno referido a problemas de sueño (i.e., "He tenido dificultad para iniciar o mantener el sueño"). Los coeficientes de fiabilidad en la presente muestra para cada una de las dimensiones del SA-45 obtenidos mediante alfa de Cronbach se incluyen en la Tabla 2.

Medida de resultados clínicos CORE-OM (Feixas et al., 2012). El CORE-OM, de sus siglas en inglés Clinical Outcomes in Routine Evaluation-Outcome Measure es un instrumento de autoinforme creado por el Core System Group (Evans, et al.,1998) para la evaluación del cambio terapéutico. Contiene 34 ítems que evalúan el malestar psicológico a partir de cuatro dimensiones: Bienestar subjetivo, Problemas/Síntomas, Funcionamiento general y Riesgo. El valor se puntúa en una escala de 0 a 4 . En el presente estudio se utilizó la versión adaptada al español (Feixas et al., 2012) y solo se incluyó la escala de Riesgo (6 ítems). Esta escala evalúa las conductas suicidas, autolesivas y de agresión e intimidación a terceros. Los estudios psicométricos realizados (Feixas et al., 2012; Trujillo et al., 2016) indican que el instrumento posee un nivel de validez y fiabilidad adecuadas, así como una excelente aceptación y sensibilidad al cambio terapéutico. El alfa de Cronbach en la muestra de estudio fue de .85

Inventario de afrontamiento COPE. Versión abreviada (Morán et al., 2010). El COPE (por sus siglas en inglés, Coping Orientation to Problems Experienced) es un inventario multidimensional, desarrollado para evaluar las diferentes formas de respuesta ante el estrés (Carver et al., 1989). Su versión breve (COPE-28; Carver, 1997) está compuesta por 28 ítems que se agrupan por pares en 14 estrategias, relativas al afrontamiento centrado en el problema (i.e., afrontamiento activo, búsqueda de apoyo instrumental, reinterpretación positiva, planificación, aceptación, renuncia y humor); afrontamiento centrado en la 
emoción (i.e., apoyo emocional y descarga emocional); afrontamiento evitativo (i.e., autodistracción, religión, negación, consumo de sustancias) y una última escala de autocrítica. Se evalúa cada una de las estrategias con una escala Likert de cuatro puntos (de 0 a 3 ) según el grado que mejor refleja la manera que el usuario tiene de enfrentarse a los problemas o dificultades en su vida: 0 "no lo refleja en absoluto"; 3 "lo refleja mucho o demasiado". En el presente estudio se utiliza la versión abreviada y adaptada al español por Morán et al. (2010). Esta versión presentó un coeficiente alfa Cronbach de .72, tanto en la versión española como en la versión inglesa (Perczek et al., 2000). En la Tabla 4 se recogen los coeficientes de fiabilidad alfa de Cronbach obtenidos en la presente muestra para cada una de las dimensiones del COPE

Escala de resiliencia de Connor-Davidson, CD-RISC (Connor y Davidson, 2003; Notario-Pacheco et al., 2011; Soler et al., 2016). La Escala de Resiliencia de Connor y Davidson (CD-RISC) fue diseñada como una herramienta para el ámbito clínico con la que poder identificar conductas resilientes y valorar las respuestas de los pacientes adultos a los tratamientos psicológicos. En su versión original el CD-RISC constaba de 25 ítems autoinformados que evaluaban cinco dimensiones: competencia personal, confianza en los propios instintos, tolerancia a la adversidad, aceptación positiva del cambio, control e influencia espiritual. Cada ítem se acompañaba de una escala Likert de cinco puntos comprendidos entre 0 "nada de acuerdo" y 4 "totalmente de acuerdo". La puntuación total proporciona una medida global de resiliencia de manera que altas puntuaciones son indicativas de alta resiliencia. Derivados de los estudios de Campbell-Stills y Stein (2007), se propone una escala unidimensional de 10 ítems con una adecuada consistencia interna (alfa de Cronbach de .85) y adecuadas evidencias de validez de constructo, convergente y discriminante. Así, Wang et al. (2010) encontraron un único factor y una buena consistencia interna (alfa de Cronbach de .91) y fiabilidad de test-retest $(r=.90$ para un intervalo de dos semanas). Sobre la versión española, Notario-Pacheco et al. (2011) hallaron adecuadas propiedades psicométricas y, en la misma línea, Serrano-Parra et al. (2013) encontraron también un factor subyacente con apropiadas validez convergente y consistencia interna (alfa de Cronbach de .81). En la presente muestra el alfa de Cronbach fue de .89 .

\section{Procedimiento}

Tras la aprobación del proyecto de investigación por parte del comité de ética de la UNED, cada terapeuta del Servicio de Psicología solicitaba la participación en el estudio a aquellos pacientes que contactaban para ser atendidos dentro del dispositivo de atención psicológica activado con motivo del estado de alarma por la COVID-19. Los terapeutas explicaban los objetivos y metodología del estudio, y solicitaban su participación voluntaria y gratuita. Una vez aceptada la participación, firmaban un consentimiento informado acorde a la protección de datos de la UNED. Todas las pruebas de evaluación se administraron a través de un procedimiento en línea idéntico para todos los participantes, utilizando la plataforma QualtricsXM( (2020). Una vez aceptada la participación los pacientes cumplimentaban los distintos instrumentos de evaluación desde su ordenador y en el orden que se ha presentado en el apartado anterior, a través de un enlace que se enviaba a su cuenta de correo electrónico. La evaluación estaba referida a la sintomatología previa a la intervención realizada. Esta evaluación se realizaba tras el primer contacto telefónico en el que se realizaba una intervención de choque dirigida a atender la demanda inicial y, en los casos que lo requería, a reducir la sintomatología en crisis. El dispositivo de intervención consistió en la aplicación de un protocolo breve de 1 a 3 sesiones acorde a una intervención en emergencias encaminado a disminuir la sintomatología inicial mediante psicoeducación y ejercicios breves de regulación. En aquellos casos en los que la persona no contaba con medios electrónicos, se ofrecía la posibilidad de cumplimentar la información solicitada mediante entrevista telefónica. En este caso, los terapeutas transcribían los datos de la entrevista telefónica a la plataforma electrónica Qualtric. El tiempo medio empleado por los participantes en cumplimentar la evaluación fue de 50 minutos. Una vez recogidos los datos se procedió a su análisis mediante el paquete estadístico IBM SPSS version 25. Durante la implementación de este dispositivo, los usuarios habituales del SPA que acudían presencialmente vieron interrumpido su tratamiento, el cual fue retomado tras la fase de confinamiento. 


\section{Análisis de datos}

Dentro de un diseño ex post facto y correlacional se analizan los datos mediante análisis descriptivo de frecuencia y porcentajes de las variables cualitativas y la obtención de medias y desviaciones tipo de las variables cuantitativas. Se analizaron las diferencias por sexo mediante Chi-cuadrado en el caso de los porcentajes y mediante análisis múltiple de la varianza en el conjunto de dimensiones pertenecientes a un mismo instrumento. Cuando los contrastes multivariados fueron significativos

Tabla 1.

Perfil de usuarios obtenido a partir de variables sociodemográficas, motivo de consulta y antecedentes $(n=124)$

\begin{tabular}{|c|c|c|c|c|c|}
\hline \multicolumn{2}{|c|}{ Perfil sociodemográfico } & \multicolumn{2}{|c|}{ Perfil de Demanda } & \multicolumn{2}{|c|}{ Perfil de Antecedentes } \\
\hline Variables & $n(\%)$ & Variables & $n(\%)$ & Variables & $n(\%)$ \\
\hline Sexo & & Ansiedad & $89(70.1)$ & Médicos & $23(18.1)$ \\
\hline Hombre & $37(29.6)$ & Estado de ánimo & $61(48.0)$ & Mentales & $39(30.7)$ \\
\hline Mujer & $88(70.4)$ & Relaciones Interp & $10(7.9)$ & Adicciones & $3(2.4)$ \\
\hline Profesión & & Pareja & $11(8.7)$ & Abuso/trauma & $9(7.1)$ \\
\hline Obrero & $6(5.0)$ & Autoestima & $12(9.4)$ & Intimidación & $6(4.7)$ \\
\hline Servicios & $18(15.3)$ & Estrés situacional & $37(29.1)$ & Viole.familiar & $12(9.4)$ \\
\hline Administrativo & $4(2.5)$ & Sueño & $32(25.2)$ & Muerte/duelo & $13(10.2)$ \\
\hline Empresario/autónomo & $2(1.7)$ & Adicciones & $1(0.8)$ & Nacimientos & $4(3.1)$ \\
\hline Técnico & $18(14.4)$ & Alimentación & $7(5.5)$ & Rupturas/pareja & $12(9.4)$ \\
\hline Ama de Casa & $9(6.8)$ & Rendimiento & $8(6.3)$ & Cambios relevte & $16(12.6)$ \\
\hline Estudiante & $36(28.0)$ & Sexuales & $2(1.6)$ & Desempleo & $13(10.2)$ \\
\hline Jubilado & $14(11.0)$ & Familiares & $26(20.5)$ & Economía & $4(3.1)$ \\
\hline Desempleado & $12(9.3)$ & Trabajo & $12(9.4)$ & Trat. Farmac & $42(33.0)$ \\
\hline Funcionario/docente & $4(2.5)$ & Somáticos & $3(2.4)$ & & \\
\hline Funcionario/no docen & $2(1.7)$ & Duelo & $10(7.9)$ & & \\
\hline Otros & $2(1.7)$ & Suicidio & $7(3.1)$ & & \\
\hline Nacionalidad & & Acoso & $4(3.1)$ & & \\
\hline Sudamericana & $12(9.5)$ & Obsesiones & $19(15.0)$ & & \\
\hline Española & $114(89.7)$ & Personalidad & $3(2.4)$ & & \\
\hline Francesa & $1(0.8)$ & Técnicas Estudio & $3(2.4)$ & & \\
\hline Estado civil & & Orientación & $1(0.8)$ & & \\
\hline Soltero & $61(48.4)$ & Discapacidad & $20(15.7)$ & & \\
\hline Casado/pareja & 47 (36.9) & Otros $^{\star}$ & (13.0) & & \\
\hline Separado/divorciado & $16(12.3)$ & & & & \\
\hline Viudo & $3(2.5)$ & & & & \\
\hline \multicolumn{6}{|l|}{ Nivel de Estudios } \\
\hline Básicos/primarios & $24(19.3)$ & & & & \\
\hline Medios & $41(32.5)$ & & & & \\
\hline Universitarios & $62(48.2)$ & & & & \\
\hline
\end{tabular}

se realizaron contrastes univariados mediante anovas tomando como factor la variable sexo y como variables dependientes, las diferentes dimensiones. Finalmente, para el análisis de la significación clínica se establecieron puntos de corte (PC) a partir de las medias (M) y desviaciones tipo (Dt) (M+/-Dt.) procedentes de las muestras publicadas (ver las referencias específicas a pie de tabla de la sección de resultados). A partir de los $\mathrm{PC}$ se hallaron los porcentajes de participantes que lo superaban $(\mathrm{PC}=\mathrm{M}+\mathrm{Dt}$., para las dimensiones del SA-45 y el CORE-OM-34) así como los porcentajes de participantes que quedaban bajo el PC (PC = M-Dt. Para el COPE-28 y el CD-RISC-10). 


\section{Resultados}

\section{Perfil sociodemográfico, perfil de demanda y antecedentes}

El perfil sociodemográfico se definió por un grupo poblacional de una edad promedio de 41.49 años $(D T=16.60)$ mayoritariamente mujeres $(70.4 \%)$, de nacionalidad española y cuyos estados civiles predominantes eran soltero/a (48.4\%) o emparejado/a (36.9\%). Las profesiones más frecuentes fueron las de estudiantes ( $28 \%)$, seguidas de las del sector servicios $(15.3 \%)$ y las técnicas (14.4\%). Mayoritariamente los usuarios tenían un nivel de estudios universitario (48.2\%) o medio (32.5\%).

El análisis de la demanda de los usuarios mostró como motivos de consulta más frecuentes, la ansiedad (70.1\%) y el bajo estado de ánimo (48 \%). En segundo lugar, el estrés situacional $(29.1 \%)$, los problemas de sueño $(25.2 \%)$ y los problemas familiares $(20.5 \%)$. Con algo más del $15 \%$, la situación de discapacidad física (e.g., motora, visual) fue también un motivo de consulta.
Entre los antecedentes personales que se relacionaron con el motivo de consulta destacaron los problemas previos de salud mental (30.7\%), así como problemas médi$\cos (18.1 \%)$ derivados de diferentes enfermedades. Con valores aproximados al $10 \%$ destacaron antecedentes relativos a los cambios en la vida cotidiana, el desempleo y la muerte de algún familiar o ser querido. Algo más del $30 \%$ de los usuarios recibía tratamiento farmacológico previo, y el $21 \%$ lo hacía de forma estable por más de tres meses. Una información más detallada sobre el conjunto de estas variables se recoge en la Tabla 1.

\section{Sintomatología y significación clínica de los usuarios}

La sintomatología y significación de las puntuaciones analizadas se muestra en la Tabla 2. Los resultados proce-

Tabla 2.

Medias (M), desviaciones tipo (Dt) y significación de la sintomatología informada por los usuarios $(n=60)$

\begin{tabular}{lcccc}
\hline & M & Dt. & Significación clínica (\%) & Alfa de Cronbach \\
\hline SA-45 & & & & \\
Hostilidad & $6.68^{*}$ & 5.50 & $40.4^{* *}$ & .81 \\
Somatización & $9.68^{*}$ & 6.52 & $59.6^{* *}$ & .86 \\
Depresión & $12.82^{*}$ & 5.84 & $82.5^{* *}$ & .85 \\
Obsesión-compulsión & $10.01^{*}$ & 5.55 & $43.9^{* *}$ & .79 \\
Ansiedad & $13.96^{*}$ & 4.87 & $86.0^{* *}$ & .80 \\
Sensibilidad Interpersonal & 9.22 & 6.07 & $63.2^{* *}$ & .84 \\
Ansiedad fóbica & $8.66^{*}$ & 7.26 & $54.4^{* *}$ & .89 \\
Ideación Paranoide & 7.85 & 5.13 & $38.6^{* *}$ & .73 \\
Psicoticismo & $4.08^{*}$ & 4.08 & $40.4^{* *}$ & .65 \\
Índice Severidad Global & $83.01^{*}$ & 36.69 & $89.5^{* *}$ & .94 \\
CORE-OM-34 & & & & - \\
Riesgo Ideación autolesiva & 1.16 & 1.59 & $24.5^{+}$ & - \\
Riesgo Planificación suicida & 0.79 & 1.37 & $14^{+}$ & -- \\
Riesgo Amenaza & 0.35 & 0.74 & $3.5^{+}$ & -- \\
Riesgo violencia & 0.61 & 1.13 & $12.3^{+}$ & -- \\
Riesgo Ideación suicida & 1.32 & 1.60 & $28.1^{+}$ & - \\
Riesgo Autolesión & 0.61 & 1.04 & $8.8^{+}$ & .85 \\
Riesgo Global & $0.80^{*}$ & 0.97 & $89.5^{* *}$ & \\
\hline
\end{tabular}

Nota: $\left(^{*}\right)$ Media que supera la puntuación normativa; Índice Severidad Global= puntuación total; $\left({ }^{* *}\right)$ Significación clínica: \% de usuarios que superan el punto de corte (media+desviación tipo) respecto de la población normativa (Sandín et al., 2008; Trujillo et al., 2016). $\left(^{+}\right)$\% de usuarios que puntuaron 3 "mucho" o 4 "extremadamente" en la escala Likert 0-4. 
Tabla 3.

Porcentaje de participantes con puntuaciones significativas en cada par de dimensiones del SA-45

\begin{tabular}{|c|c|c|c|c|c|c|c|c|c|}
\hline & 1 & 2 & 3 & 4 & 5 & 6 & 7 & 8 & 9 \\
\hline 1. Hostilidad & -- & 72.40 & 65 & 72.4 & 92.6 & 62.1 & 56.2 & 74.4 & 96.1 \\
\hline 2. Somatización & & -- & 69.8 & 65.7 & 100 & 69.6 & 80 & 54.3 & 57.1 \\
\hline 3.Depresión & & & - & 72.1 & 95.3 & 51.2 & 67.4 & 58.1 & 51.2 \\
\hline 4.Obsesión-compulsión & & & & - & 63.3 & 78.3 & 71.9 & 65.6 & 71.4 \\
\hline 5.Ansiedad & & & & & - & 95.7 & 69.4 & 49 & 44.9 \\
\hline 6.Sensibilidad Interpersonal & & & & & & -- & 95.6 & 91.7 & 96.1 \\
\hline 7.Ansiedad fóbica & & & & & & & - & 51.4 & 64.3 \\
\hline 8.Ideación Paranoide & & & & & & & & -- & 71.4 \\
\hline 9.Psicoticismo & & & & & & & & & - \\
\hline
\end{tabular}

dentes del listado de síntomas SA-45 mostraron elevadas puntuaciones en la mayoría de los síndromes evaluados así como en el nivel de gravedad global. Consistentemente con los motivos de consulta, las dimensiones que presentaron niveles más altos de sintomatología fueron las correspondientes a ansiedad, depresión y somatización. Todas las dimensiones evaluadas, con excepción de las referidas a sensibilidad interpersonal y psicoticismo, puntuaron por encima de los valores obtenidos en muestras normativas. La significación clínica o gravedad de la sintomatología estimada mediante el porcentaje de usuarios de la muestra que superaban el punto de corte establecido osciló entre el $38.6 \%$ en la dimensión de ideación paranoide y el $86 \%$ de la de ansiedad. De nuevo, los porcentajes más elevados de usuarios que superaron el punto de corte se obtuvo en las dimensiones de Ansiedad, Depresión y Somatización. La sensibilidad interpersonal, a pesar de que la media grupal no fue diferente de la media normativa, resultó superior al punto de corte establecido en un elevado porcentaje de usuarios. Considerando el nivel global de gravedad obtenido por la puntuación total de las diferentes escalas, aproximadamente el $90 \%$ de la muestra superaba los niveles normativos. En la misma línea se mostró la dimensión de riesgo evaluada mediante el COREOM que evalúa la presencia de conductas suicidas, autolesivas y de agresión e intimidación a terceros. La media obtenida por la muestra del presente estudio resultó superior a la media normativa y casi el $90 \%$ de los usuarios superaba el punto de corte establecido. Cabe destacar que más del $20 \%$ de la muestra estudiada mostró ideación autolesiva e ideación suicida con niveles informados de "altos" o "muy altos".
Adicionalmente a estas dimensiones se evaluaron los niveles de rumiación y los problemas de sueño. Aunque estas puntuaciones no se pudieron comparar con muestras normativas, los usuarios informaron de elevados niveles tanto de rumiación así como de problemas de sueño. Respecto a los pensamientos rumiativos el $26.4 \%$ y el $35.1 \%$ puntuaron respectivamente y con los niveles más altos de la escala de medida, "sentir preocupación y ansiedad por muchas cosas y no poder controlarlo" y "Me preocupo en exceso por muchas cosas como la salud, familia, economía, trabajo... y no puedo controlarlo" De manera similar ocurrió con los niveles informados relativos a los problemas de sueño: el $65 \%$ informó tener de manera muy frecuente dificultades para conciliar o mantener el sueño.

El análisis de la comorbilidad (Tabla 3) puso de manifiesto porcentajes de sintomatología comórbida significativa que oscilaba entre el $44.9 \%$ (i.e., ansiedad y psicoticismo) y el $100 \%$ (i.e., somatización y ansiedad) de los participantes. Destacó la comorbilidad entre los síntomas de ansiedad y somatización (100\%), sensibilidad interpersonal con psicoticismo, ansiedad e ideación paranoide (respectivamente $96.1 \%, 95.7 \%, 91.7 \%$ ), hostilidad con psicoticismo y ansiedad (respectivamente $96.1 \%, 92.6 \%$ ) y depresión y ansiedad (95.3\%).

\section{Estrategias de afrontamiento y resiliencia}

Las estrategias de afrontamiento más frecuentemente utilizadas por los usuarios fueron las de búsqueda de 
Tabla 4.

Medias, desviaciones tipo y significación de las estrategias de afrontamiento y nivel de resiliencia percibida ( $n=60)$

\begin{tabular}{|c|c|c|c|c|}
\hline & Media & Desviación tipo & Significación clínica (\%) & Alfa de Cronbach \\
\hline \multicolumn{5}{|l|}{ COPE-28 } \\
\hline Afrontamiento Activo & $3.49^{*}$ & 1.65 & 70.2 & .65 \\
\hline Planificación & $2.82^{*}$ & 1.67 & 82.5 & .44 \\
\hline Reinterpretación positiva & $2.40^{*}$ & 1.68 & 73.7 & .64 \\
\hline Aceptación & $2.85^{*}$ & 1.76 & 61.4 & .58 \\
\hline Humor & $1.42^{*}$ & 1.63 & 59.6 & .61 \\
\hline Religión & 1.82 & 2.17 & 17.5 & .81 \\
\hline Apoyo emocional & $3.10^{*}$ & 1.81 & 68.4 & .63 \\
\hline Apoyo social & $3.52^{*}$ & 1.84 & 42.1 & .68 \\
\hline Autodistracción & $2.64^{*}$ & 1.74 & 70.2 & .48 \\
\hline Negación & 1.80 & 2.01 & 86.0 & .64 \\
\hline Desahogo & $2.35^{\star}$ & 1.76 & 73.7 & .50 \\
\hline Uso de sustancias & $1.21^{*}$ & 1.97 & 68.4 & .88 \\
\hline Desconexión & $1.84^{*}$ & 1.91 & 68.4 & .71 \\
\hline Autoculpa & 3.08 & 2.20 & -- & 69 \\
\hline \multicolumn{5}{|l|}{ CD-RISC-10 } \\
\hline Resilencia & $18.28^{*}$ & 9.69 & 66.7 & .89 \\
\hline $\begin{array}{l}\text { Nota: }\left({ }^{*}\right) \text { Media inferior al } \\
\text { Perczek et al., 2000). } \\
\text { (**) Significación clínica: } \\
\text { respecto de la población } \\
\text { et al., 2011: Soler et al., }\end{array}$ & a c & a menos u & viación tipo) de la muest & $\begin{array}{l}\text { (Luzdivina, 2015; } \\
\text { desviación tipo) } \\
-10 \text { (Notario-Pache }\end{array}$ \\
\hline
\end{tabular}

apoyo (Apoyo social y emocional), Afrontamiento activo y Autoculpa. En segundo lugar, con niveles ligeramente inferiores, las estrategias de Aceptación, Planificación y Autodistracción (Tabla 4). Los niveles de frecuencia de la mayoría de las estrategias de afrontamiento resultaron inferiores a las informadas por la población normativa, exceptuando las de Religión y Negación que resultaron similares a los niveles normativos. Los porcentajes de usuarios que utilizaban deficitariamente estas estrategias oscilaron entre el $17.5 \%$ en la estrategia de Religión hasta el $82.5 \%$ en la estrategia de Planificación, con valores entre el 60$70 \%$ en un gran número de ellas. En sentido positivo, un gran porcentaje de usuarios no utilizó de manera frecuente estrategias desadaptativas como la Negación $(86 \%)$ o el Consumo de sustancias (68.4\%). Tomando el conjunto de estos resultados, los datos muestran en general un uso deficitario de estrategias de afrontamiento positivo.

En la misma dirección de estos resultados aparecieron los niveles de resiliencia informado con una puntuación media inferior a la media normativa. Más del $65 \%$ de los participantes mostraron niveles de resiliencia inferiores al punto de corte estimado.

\section{Diferencias por sexo en las variables estudiadas}

En relación con las características sociodemográficas, tipo de demanda y antecedentes analizados la distribución de las variables resultó estadísticamente similar entre hombres y mujeres tal y como mostraron las comparaciones de porcentajes mediante Chi-cuadrado $(p<.05)$ en cada una de ellas.

El análisis de las diferencias de sexo en la sintomatología analizada se llevó a cabo mediante análisis múltiple de la varianza (MANOVA). Los resultados indicaron la ausencia de un efecto significativo multivariado para la variable sexo (Wilks' Lambda $=.75, \quad \mathrm{~F}_{(9,50)}=1.57$, $\left.p=.15, \eta_{\mathrm{p}}^{2}=.24\right)$. No obstante, las comparaciones univariadas entre hombres y mujeres en cada una de las dimen- 
siones mostró un efecto significativo de la variable sexo para la sintomatología depresiva $\mathrm{F}_{(1,58)}=5.72$; $\left.\mathrm{MCe}=186.80 ; \quad p=.020 ; \quad \eta_{\mathrm{p}}^{2}=.10\right) . \quad$ Las mujeres $(M=14.02, D T=0.93)$ mostraron niveles de sintomatología depresiva significativamente más altos que los hombres $(M=9.93, D T=1.42)$. Adicionalmente, las diferencias por sexo en la dimensión de riesgo global, rumiaciones y problemas de sueño no resultaron significativas.

Respecto a las diferencias por sexo en las estrategias de afrontamiento y resiliencia informadas por los participanes, los resultados no mostraron diferencias significativas ni en el análisis multivariado (Wilks' Lambda $=.80$, $\left.\mathrm{F}_{(14,45)}=0.66, p=.78, \eta_{\mathrm{p}}^{2}=.19\right)$ ni en el univariado $(p<.05)$.

\section{Discusión}

El objetivo general de esta investigación era analizar la atención psicológica gratuita ofrecida desde el dispositivo puesto en marcha por el Servicio de Psicología Aplicada de la UNED, como consecuencia del estado de alarma por la COVID-19. Por lo que se refiere al perfil sociodemográfico de los solicitantes del servicio, los usuarios tenían una edad media de 40 años, era principalmente de nacionalidad española y, en línea con la literatura (Casagrande et al., 2020; Favieri et al., 2020; Qiu et al., 2020; Rodríguez-Rey et al., 2020), mayoritariamente mujeres. El estado civil predominante fue el de soltero/a, no obstante, un número importante vivía en pareja. Por lo que se refiere a la situación laboral, sólo una minoría tenían la condición de desempleado pero el grupo formado por estudiantes, amas de casa y jubilados (condiciones sin retribución o bajos ingresos económicos) supusieron casi la mitad de la población. En parte, los resultados fueron congruentes con aquellos estudios que muestran que estar en pareja y tener empleo son factores protectores para la aparición de sintomatología por confinamiento (Li y Wang, 2020). En relación con la formación, aunque otros estudios señalan el bajo nivel de estudios como un factor de riesgo (Fancourt et al., 2021), los participantes de este estudio tenían mayoritariamente un nivel educativo medio o universitario. Tanto el porcentaje de estudiantes como su perfil formativo pueden explicarse por el contexto universitario en el que se ubica el SPA-UNED en el que tuvo lugar la investigación.

Los problemas mentales seguidos de las dificultades de salud física fueron los antecedentes de mayor prevalencia entre los usuarios, dato congruente con otros estudios (Mak et al., 2009). Casi un tercio de los usuarios recibían tratamiento farmacológico y un $21 \%$ lo hacía de manera estable con una duración de al menos tres meses, lo que indica la presencia de problemas de salud previos como distintivo de vulnerabilidad. Así mismo y aunque en menor medida, destacaron los antecedentes relacionados con la pérdida de un ser querido, circunstancia que se vio incrementada por el número de fallecimientos por COVID19.

En cuanto al contenido de las demandas, los usuarios referían principalmente ansiedad, percepción de un bajo estado de ánimo relacionado con la situación, lo que resulta consistente con lo encontrado de manera sistemática en estudios con población española (Ozamiz-Etxebarria et al., 2020; Sandín et al., 2020) y extranjera (e.g., Fancourt et al., 2021; Qiu et al., 2020; Serafini et al. 2020). Gran parte de estas demandas derivaban, como han señalado otros estudios (Favieri et al., 2020; Li y Wang, 2020; Taquet et al., 2021), de la situación vivida por el confinamiento. También y en sintonía con otros estudios (Casagrande et al., 2020; Ozamiz-Etxebarria et al., 2020; Sandín et al., 2020), la percepción de estrés fue otro de los motivos frecuentes de búsqueda de ayuda psicológica. Además, un tercio de los usuarios señalaron problemas de sueño, sintomatología encontrada por otros autores (Casagrande et al., 2020; Torales et al., 2020).

El análisis de la sintomatología y su significación clínica, al igual que lo encontrado por otros autores (Favieri et al., 2020; Li y Wang, 2020; Rodríguez-Rey et al., 2020; Sandín et al., 2020) constató un impacto psicológico negativo del confinamiento en el bienestar psicológico de los usuarios caracterizado por elevados niveles de ansiedad, depresión y somatización. Además, un porcentaje elevado de los usuarios informaron de elevada sensibilidad interpersonal (i.e., incomprensión, enemistad o rechazo, inferioridad) y sintomatología paranoide (i.e., pensamiento autorreferencial, de control ajeno, o suspicacia). Por último, casi la totalidad superaron los niveles promedios 
normativos de los diferentes síndromes, lo que resultaba esperable tratándose de personas que buscan ayuda en un servicio de atención psicológica. Un dato a destacar, es la elevada sintomatología comórbida que presentaron los usuarios, más del 90\% de los participantes informó de comorbilidad entre las dimensiones de somatización- ansiedad, depresión- ansiedad, sensibilidad interpersonal con psicoticismo, ansiedad e ideación paranoide y hostilidad con psicoticismo y ansiedad.

Para completar el análisis del ajuste y el bienestar psicológicos se evaluó la presencia de conductas autolesivas y de intimidación, el pensamiento rumiativo y las dificultades de sueño. Respecto al comportamiento autolesivo e intimidatorio, la totalidad de los usuarios estudiados informaron de niveles clínicamente significativos y casi un tercio de ellos mostraban niveles altos o muy altos de ideación autolesiva y suicida. A este respecto, en la literatura se alerta del riesgo del incremento de conductas suicidas (Reger, et al., 2020) y de la relación entre el confinamiento y estas conductas (Brooks et al. 2020, Thombs et al., 2020). Respecto de la rumiación, más de un tercio de la muestra estudiada informó de niveles altos de pensamientos repetitivos y más de la mitad de problemas de sueño sintomatologías congruentes tanto con el bajo estado de ánimo como con la ansiedad previamente informadas.

En relación con las estrategias de afrontamiento y el nivel de resiliencia de los usuarios, los resultados mostraron un uso deficitario de las diferentes estrategias de afrontamiento respecto de la población normativa, con excepción de la negación y la religión, que resultaron similares a los niveles normativos (Luzdivina, 2015; Perczek et al., 2000). De todas estas estrategias, las más utilizadas $\mathrm{y}$, a la vez, deficitarias, fueron la de búsqueda de apoyo social y emocional, el afrontamiento activo y la autoculpa, seguidas de estrategias de aceptación, planificación y autodistracción. El déficit de un adecuado afrontamiento es congruente con la sintomatología hallada y puede ser una de las razones que junto con la amenaza percibida del estresor (i.e., pandemia) la explique. En línea con estos resultados, se mostraron niveles de resiliencia por debajo de la población normativa (Notario-Pacheco et al., 2011; Soler et al., 2016).
En cuanto a las diferencias por sexo en los distintos perfiles (i.e., sociodemográfico, sindrómico y de afrontamiento), los usuarios fueron principalmente mujeres, quienes además, presentaron niveles significativamente superiores de sintomatología depresiva respecto de los hombres. Está ampliamente avalado por la investigación los niveles más altos de sintomatología interiorizada, particularmente en ansiedad y depresión en las mujeres frente a los hombres, y en relación con las experiencias de pandemia (i.e., Qiu et al., 2020; Rodríguez-Rey et al., 2020). No obstante, en la muestra estudiada ni los niveles de resiliencia ni las estrategias de afrontamiento resultaron diferentes entre hombres y mujeres.

El conjunto de estos resultados es compatible con un contexto de elevado estrés poblacional generalizado y confinamiento y, más aún, entre personas más vulnerables, a juzgar por sus antecedentes personales, su inadecuado afrontamiento y sus bajos niveles de resiliencia. Los resultados son congruentes con alteraciones adaptativas relacionadas a factores de estrés en los que predomina la sintomatología de ansiedad y estado de ánimo deprimido (APA, 2013) que pueden muy bien ser explicadas por los modelos generales de estrés.

Aunque, como hemos visto, los resultados obtenidos se encuentran en sintonía con los encontrados por investigaciones previas, la presente investigación tiene algunas limitaciones a considerar. En primer lugar, se trata de una muestra de estudio reducida e incidental que restringe la representación poblacional y la generalización de los resultados a la población clínica. En segundo lugar, las variables objeto de estudio se han evaluado mediante autoinformes, primando el carácter subjetivo de las medidas frente a indicadores intersubjetivos procedentes de una evaluación multimétodo o multi-informante. No obstante, a pesar de las limitaciones expuestas, nuestro estudio se llevó a cabo en condiciones de especial dificultad (un estado de alerta sanitaria bajo confinamiento y alto riesgo de contagio) que hacen que estos resultados puedan contribuir a un mayor y mejor conocimiento de las variables psicológicas personales y grupales que, a su vez, permitan la implementación de actuaciones preventivas sobre los perfiles de riesgo y vulnerabilidad identificados. 


\section{Referencias}

American Psychiatric Association. (2013). Diagnostic and Statistical Manual of Mental Disorders (DSM$5 \circledR)$. Autor.

Araujo, M., García, S. y García-Navarro, E. B. (2020). Abordaje del duelo y de la muerte en familiares de pacientes con COVID-19: Revisión narrativa [Approaching grief and Death in Family Members of Patients with COVID-19: Narrative Review]. Enfermeria Clinica, 31, S112-S116. https://doi.org/10.1016/j.enfcli.2020.05.011

Balluerka, N., Gómez, J., Hidalgo, M. D., Gorostiaga, A., Espada, J. P., Padilla, J. L. y Santed, M. A. (2020). Las consecuencias psicológicas de la COVID-19 y el confinamiento [The Psychological Consequences of COVID-19 and Confinement] [Informe de investigación]. Universidad del País Vasco.

https://www.ciencia.gob.es/stfls/MICINN/Univers idades/Ficheros/Consecuencias_psicologicasCOVI D19.pdf

Brooks, S. K., Webster, R. K., Smith, L. E., Woodland, L., Wessely, S., Greenberg, N. y Rubin, G. J. (2020). The Psychological Impact of Quarantine and how to Reduce it: Rapid Review of the Evidence. The Lancet, 395(10227), 912-920. https://doi.org/10.1016/S0140-6736(20)30460-8

Cabrera, E. A. (2020). Actividad física y efectos psicológicos del confinamiento por COVID-19 [Physical Activity and Psychological Effects of Confinement by COVID-19]. Revista INFAD de Psicología. International Journal of Developmental and Educational Psychology, 2(1), 209-220.

https://doi.org/10.17060/ijodaep.2020.n1.v2.1828

Casagrande, M., Favieri, F., Tambelli, R. y Forte, G. (2020). The Enemy who Sealed the World: Effects Quarantine Due to the COVID-19 on Sleep Quality, Anxiety, and Psychological Distress in the Italian population. Sleep Medicine, 75, 12-20. https://doi.org/10.1016/j.sleep.2020.05.011

Conversano, C., Marchi, L. y Miniati, M. (2020). Psychological Distress among Healthcare Professionals Involved in the COVID-19 emergency: Vulnerability and Resilience Factors. Clinical Neuropsychiatry: Journal of Treatment Evaluation, 17(2), 94-96. https://doi.org/10.36131/CN20200212

Connor, K. M. y Davidson, J. R. (2003). Development of a new Resilience Scale: The Connor-Davidson Resilience Scale (CD-RISC). Depress Anxiety, 18(2), 76-82. https://doi.org/10.1002/da.10113

Czeisler, M. E., Lane, R. I., Petrosky, E., Wiley, J. F., Christensen, A., Njai, R., Weaver, M. D., Robbins, R., Facer-Childs, E. R., Barger, L. K., Czeisler, C. A., Howard, M. E. y Rajaratnam, S. M. W. (2020). Mental Health, Substance Use, and Suicidal Ideation During the COVID-19 Pandemic-United States, June 24-30, 2020. Morbidity and Mortality Weekly Report, 69(32), 1049-1057. https://doi.org/10.15585/mmwr.mm6932a1

Dosil, M., Ozamiz-Etxebarria, N., Redondo, I., Jaureguizar, J. y Picaza, M. (2020). Impacto psicológico de la COVID-19 en una muestra de profesionales sanitarios españoles [Psychological impact of COVID-19 on a Simple of Spanish Health Professionals]. Revista de Psiquiatría y Salud Mental, 14, 106-112. https://doi.org/10.1016/j.rpsm.2020.05.004

Fancourt, D., Steptoe, A. y Bu, F. (2021). Trajectories of Anxiety and Depressive Symptoms during Enforced Isolation Due to COVID-19 in England: A Longitudinal Observational Study. The Lancet Psychiatry, 8(2), 141-149. https://doi.org/10.1016/S2215-0366(20)30482-X

Favieri, F., Forte, G., Tambelli, R., y Casagrande, M. (2021). The Italians in the Time of Coronavirus: Psychosocial Aspects of the Unexpected COVID- 
19 Pandemic. Frontiers in Psychiatry, 12, Artículo 551924. https://doi.org/10.3389/fpsyt.2021.551924

Feixas, G., Evans, C., Trujillo, A., Saúl, L. A., Botella, L., Corbella, S., González, E., Bados, A, GarcíaGrau, E. y López-González, M. A. (2012). La versión española del CORE-OM: Clinical Outcomes in Routine Evaluation - Outcome Measure [Spanish Version of the Clinical Outcomes in Routine Evaluation-Outcome Measure (CORE-OM)]. Revista de Psicoterapia, 23(89), 109-135.

Garfin, D. R., Silver, R. C. y Holman, E. A. (2020). The Novel Coronavirus (COVID-2019) Outbreak: Amplification of Public Health Consequences by Media Exposure. Health Psychology, 39(5), 355357. https://doi.org/10.1037/hea0000875

Kim, S.-W. y Su, K.-P. (2020). Using Psychoneuroimmunity against COVID-19. Brain, Behavior, and Immunity, 87, 4-5. https://doi.org/10.1016/j.bbi.2020.03.025

Li, L. Z. y Wang, S. (2020). Prevalence and Predictors of General Psychiatric Disorders and Loneliness during COVID-19 in the United Kingdom. Psychiatry Research, 291, Artículo 113267. https://doi.org/10.1016/j.psychres.2020.113267.

Morán, C., Landero, R. y González, M. T. (2010). COPE-28: un análisis psicométrico de la versión en español del Brief COPE [COPE-28: A Psychometric Analysis of the Spanish Version of the Brief COPE]. Universitas Psychologica, 9(2), 543-552.

Mortazavi, S. S., Assari, S., Alimohamadi, A., Rafiee, M. y Shati, M. (2020). Fear, Loss, Social Isolation, and Incomplete Grief Due to COVID-19: A Recipe for a Psychiatric Pandemic. Basic and Clinical Neuroscience, $\quad 11(2), \quad 225-232$. https://doi.org/10.32598/bcn.11.COVID19.2549.1

Notario-Pacheco, B., Solera, M., Serrano, M. D., Bartolomé, R.,García-Campayo, J. y Martínez-
Vizcaíno, V. (2011). Reliability and Validity of the Spanish Version of the 10 item Connor-Davidson Resilience Scale (10 item CDRISC) in Young Adults. Health Quality Life Outcomes, 9, 63-68. https://doi.org/10.1186/1477-7525-9-63

Organización Mundial de la Salud. (2021). WHO Coronavirus Disease (COVID-19) Dashboard. https://COVID19.who.int

Ozamiz-Etxebarria, N., Idoiaga, N., Dosil, M. y Picaza, M. (2020). Psychological Symptoms During the Two Stages of Lockdown in Response to the COVID-19 Outbreak: An Investigation in a Sample of Citizens in Northern Spain. Frontiers in Psychology, 11, Artículo 1491. https://doi.org/10.3389/fpsyg.2020.01491

Qiu, J., Shen, B., Zhao, M., Wang, Z., Xie, B. y Xu, Y. (2020). A Nationwide Survey of Psychological Distress among Chinese People in the COVID-19 Epidemic: Implications and Policy Recommendations. General Psychiatry, 33(2), Artículo e100213. https://doi.org/10.1136/gpsych2020-100213

Reger, M. A., Stanley, I. H. y Joiner, T. E. (2020). Suicide Mortality and Coronavirus Disease 2019. A Perfect Storm? JAMA Psychiatry, 77(11), 10931094.

https://doi.org/10.1001/jamapsychiatry.2020.1060

Rodríguez-Rey, R., Garrido-Hernansaiz, H. y Collado, S. (2020). Psychological Impact and Associated Factors During the Initial Stage of the Coronavirus (COVID-19) Pandemic Among the General Population in Spain. Frontiers in Psychology, 11, Artículo 1540. https://doi.org/10.3389/fpsyg.2020.01540

Sánchez-Villena, A. R. y de La Fuente-Figuerola, V. (2020). COVID-19: Cuarentena, aislamiento, distanciamiento social y confinamiento, ¿son lo mismo? [COVID-19: Quarantine, Isolation, Social Distancing and Lock Down: Are they the Same?]. 
Anales de Pediatría, 93(1), 73-74. https://doi.org/10.1016/j.anpedi.2020.05.001

Sandín, B., Valiente, R. M., Chorot, P., Santed, M. A. y Lostao, L. (2008). SA-45: Forma abreviada del SCL-90 [SA-45: A brief form of the SCL-90]. Psicothema, 20(2), 290-296.

Sandín, B., Valiente, R. M., García-Escalera, J. y Chorot, P. (2020). Impacto psicológico de la pandemia de COVID-19: Efectos negativos y positivos en población española asociados al periodo de confinamiento nacional [Psychological impact of the COVID-19 Pandemic: Negative and Positive Effects in Spanish People during the Mandatory National Quarantine]. Revista de Psicopatología y Psicología Clínica, 25(1), 1-22. https://doi.org/10.5944/rppc.27569

Serafini, G., Parmigiani, B., Amerio, A., Aguglia, A., Sher, L. y Amore, M. (2020). The Psychological Impact of COVID-19 on the Mental Health in the General Population. QJM: An International Journal of Medicine, 113(8), 531-537. https://doi.org/10.1093/qjmed/hcaa201

Soler, M. I., Meseguer, M. y García, M. (2016). Propiedades psicométricas de la versión española de la escala de resiliencia de 10 ítems de ConnorDavidson (CD-RISC 10) en una muestra multiocupacional [Psychometric Properties of the Spanish Version of the 10-item Connor-Davidson Resilience Scale (10-item CD-RISC) in a Sample of Workers]. Revista Latinoamericana de Psicología, 48(3), 159-166. https://doi.org/10.1016Zj.rlp.2015.09.002

Szcześniak, D., Gładka, A., Misiak, B., Cyran, A. y Rymaszewska, J. (2021). The SARS-CoV-2 and Mental Health: From Biological Mechanisms to Social Consequences. Progress in NeuroPsychopharmacology and Biological Psychiatry, 104, Artículo 110046. https://doi.org/10.1016/j.pnpbp.2020.110046
Taquet, M., Luciano, S., Geddes, J. R. y Harrison, P. J. (2021). Bidirectional Associations between COVID-19 and Psychiatric Disorder: Retrospective Cohort Studies of 62354 COVID-19 Cases in the USA. The Lancet Psychiatry, 8(2), 130-140. https://doi.org/10.1016/S2215$\underline{0366(20) 30462-4}$

Thombs, B. D., Bonardi, O., Rice, D. B., Boruff, J. T., Azar, M., He, C., Markham, S., Sun, Y., Wu, Y. y Krishnan, A. (2020). Curating Evidence on Mental Health during COVID-19: A Living Systematic Review. Journal of Psychosomatic Research, 133, Artículo 110113. https://doi.org/10.1016/j.jpsychores.2020.110113

Torales, J., O'Higgins, M., Castaldelli-Maia, J. M. y Ventriglio, A. (2020). The Outbreak of COVID-19 Coronavirus and its Impact on Global Mental Health. International Journal of Social Psychiatry, 66(4), 317-320. https://doi.org/10.1177/0020764020915212

Trujillo, A., Feixas, G., Bados, A., García-Grau, E., Salla, M., Medina, J. C., Montesano, A., Soriano, J., Medeiros-Ferreira, L., Cañete, J., Coreblla, S., Grau, A., Lana, F. Y Evans, C. (2016). Psychometric Properties of the Spanish Version of the Clinical Outcomes in Routine EvaluationOutcome Measure. Neuropsychiatric Disease and Treatment, 12, 1457-1466. https://doi.org/10.2147/NDT.S103079

Wallace, C. L., Wladkowski, S. P., Gibson, A. y White, P. (2020). Grief During the COVID-19 Pandemic: Considerations for Palliative Care Providers. Journal of Pain and Symptom Management, 60(1), e70-e76.

https://doi.org/10.1016/j.jpainsymman.2020.04.01 $\underline{2}$

Wilder-Smith, A. y Freedman, D. O. (2020). Isolation, Quarantine, Social Distancing and Community Containment: Pivotal Role for Old-Style Public Health Measures in the Novel Coronavirus (2019nCoV) Outbreak. Journal of Travel Medicine, 
Artículo

taaa020.

https://doi.org/10.1093/jtm/taaa020

Wu, K. K., Chan, S. K. y Ma, T. M. (2005).

Posttraumatic Stress, Anxiety, and Depression in Survivors of Severe Acute Respiratory Syndrome (SARS). Journal of Traumatic Stress, 18(1), 39-42.

https://doi.org/10.1002/jts.20004 
